\title{
Noise-to-state exponential stability of neutral random nonlinear systems
}

Wen Lu ${ }^{1 *}$

"Correspondence: Ilcxw@163.com

'School of Mathematics and Informational Science, Yantai

University, Yantai, China

\begin{abstract}
In this paper, we deal with a class of neutral random nonlinear systems. The existence and uniqueness of the solution to neutral random functional nonlinear systems (NRFSs) are established. Furthermore, the criteria on noise-to-state stability in the moment of a special class of NRFSs, named neutral random delay nonlinear systems, are derived. An example is given for illustration.
\end{abstract}

MSC: Primary 34K50; secondary 93E15; 60H10

Keywords: Neutral random nonlinear system; Second-order stationary process; Noise-to-state exponential stability

\section{Introduction}

Stochastic differential equations (SDEs) are widely adopted to describe systems with stochastic disturbances. As one of the most important issues of SDEs, stability theory of SDEs has been studied intensively over past decades, see for instance [1-6] and the references therein.

Moreover, delay effects are common phenomena which exist in a wide variety of real systems. Neutral differential equations with delay (NDDEs in short) are often used to describe the systems which depend not only on present and past states but also involve derivatives with delays. For theory of NDDEs, one can see Hale and Lunel [7] and the references therein. Taking the environmental disturbances into account, Kolmanovskii and Nosov [8] and Mao [9] discussed the neutral stochastic differential equations with delay driven by Brownian motion (NSDDEs). Since then, so many efforts have been made on this topic, especially on asymptotical boundedness and exponential stability of NSDDEs. One can see $[10-13]$ and the references therein.

Although fruitful results on the stability of SDEs have been obtained, it seems that the model of SDEs is not necessarily the best model in some specific situations (see $[14,15]$ for more details). For example, stationary processes are more reasonable to describe stochastic disturbances to other electric elements in circuit systems with power noise filter than white noises. Stationary processes have been applied to automatic control, information theory, and wireless technology. In $\mathrm{Wu}[14]$, a framework of stability analysis for random nonlinear systems with second-order processes was established. The notion of noise-tostate stability was proposed for stochastic nonlinear systems by regarding the unknown

(c) The Author(s) 2018. This article is distributed under the terms of the Creative Commons Attribution 4.0 International License (http://creativecommons.org/licenses/by/4.0/), which permits unrestricted use, distribution, and reproduction in any medium, provided you give appropriate credit to the original author(s) and the source, provide a link to the Creative Commons license, and indicate if changes were made. 
covariance of Brownian motion as the deterministic input in [16] and [17]. Based on the above works, Zhang et al. [18] investigated the noise-to-state stability in probability of state-dependent switched random nonlinear systems with second-order stationary processes. Then, Zhang et al. [15] and Zhang et al. [19] established the criteria on noise-tostate stability in the moment of random switched systems under a reasonable assumption of second-order processes, respectively.

In this paper, motivated by the above-mentioned works, we deal with a class of neutral random functional nonlinear systems of the form

$$
d\left[x(t)-D\left(x_{t}\right)\right]=f\left(x_{t}, t\right) d t+g\left(x_{t}, t\right) \xi(t) d t, \quad t \geq t_{0}
$$

with initial data $x_{t_{0}}$. Here, the same as in [14], $\xi(t)$ is a second-order process. The existence and uniqueness of the solution to system (1) and the noise-to-state exponential stability of an important class of system (1) are considered. We mentioned that, when $\xi(t)$ is a white noise, regarding $\xi(t)$ as the formal derivative of a Brownian motion (or Wiener process) $B(t)$, equation (1) becomes the classical functional SDE described by

$$
d\left[x(t)-D\left(x_{t}\right)\right]=f\left(x_{t}, t\right) d t+g\left(x_{t}, t\right) d B(t), \quad t \geq t_{0}
$$

with initial data $x_{t_{0}}$.

The paper is organized as follows. In Sect. 2, we introduce some notations and preliminaries. Section 3 is devoted to the existence and uniqueness of solution to a neutral random functional nonlinear system. In Sect. 4, the criteria of noise-to-state exponential stability in the $p$ th moment of neutral random delay nonlinear systems are established. An example is given in Sect. 5 to illustrate our main results.

\section{Notations and preliminaries}

Throughout this paper, unless otherwise specified, we use the following notations. Let $|\cdot|$ be the Euclidean norm in $R^{n}$. If $A$ is a vector or matrix, its transpose is denoted by $A^{T}$. If $A$ is a matrix, its trace norm is denoted by trace $|A|=\sqrt{\operatorname{trace}\left(A^{T} A\right)}$. Let $\tau>0$ and denote by $C\left([-\tau, 0] ; R^{n}\right)$ the family of continuous functions $\varphi$ from $[-\tau, 0]$ to $R^{n}$ with the norm $\|\varphi\|=\sup _{-\tau \leq \theta \leq 0}|\varphi(\theta)|$. Let $R_{+}=[0, \infty)$ and $\mathcal{K}$ stands for the set of all functions $R_{+} \rightarrow R_{+}$, which are continuous, strictly increasing, and vanishing at zero.

Let $\left(\Omega, \mathcal{F}, \mathcal{F}_{t}, P\right)$ be a given complete probability space with a filtration $\left\{\mathcal{F}_{t}\right\}_{t \geq 0}$ satisfying the usual conditions. For $p>0$, let $\mathcal{L}_{\mathcal{F}_{t_{0}}}^{p}\left([-\tau, 0] ; R^{n}\right)$ be the family of $\mathcal{F}_{t_{0}}$-measurable $C\left([-\tau, 0] ; R^{n}\right)$-valued random variables $\eta$ such that $E\|\eta\|^{p}<\infty$. Denote by $C_{\mathcal{F}_{t_{0}}}^{b}\left([-\tau, 0] ; R^{n}\right)$ the family of all $\mathcal{F}_{t_{0}}$-measurable, bounded, and $C\left([-\tau, 0] ; R^{n}\right)$-valued random variables.

Consider a neutral random functional nonlinear system of the form

$$
d\left[x(t)-D\left(x_{t}\right)\right]=f\left(x_{t}, t\right) d t+g\left(x_{t}, t\right) \xi(t) d t, \quad t \geq t_{0}
$$

with initial data $x_{t_{0}}=\eta=\{\eta(\theta):-\tau \leq \theta \leq 0\} \in \mathcal{L}_{\mathcal{F}_{t_{0}}}^{1}\left([-\tau, 0] ; R^{n}\right)$, where $D: C\left([-\tau, 0] ; R^{n}\right) \rightarrow$ $R^{n}, f: C\left([-\tau, 0] ; R^{n}\right) \times R^{+} \rightarrow R^{n}$ and $g: C\left([-\tau, 0] ; R^{n}\right) \times R^{+} \rightarrow R^{n \times d}$ are Borel-measurable, $x(t) \in R^{n}$ is the state of the system and $x_{t}=\{x(t+\theta):-\tau \leq \theta \leq 0\}$ is regarded as a 
$C\left([-\tau, 0] ; R^{n}\right)$-valued stochastic process. The stochastic process $\xi(t) \in R^{d}$ is a secondorder process satisfying the following assumption.

Assumption A1 The process $\xi(t) \in R^{d}$ is $\mathcal{F}_{t}$-adapted and piecewise continuous and satisfies

$$
\sup _{t_{0} \leq s \leq t} E|\xi(s)|^{2}<\infty, \quad \forall t \geq t_{0}
$$

Remark 1 From $\sup _{t_{0} \leq s \leq t} E|\xi(s)|^{2}<\infty$, for all $t \geq t_{0}$, one can verify $\xi(t)<\infty$, a.s.

Definition 1 A solution to system (2) on $t \geq t_{0}-\tau$ is a process $x(t):=x\left(t ; t_{0}, x_{t_{0}}\right)$ satisfying the following:

1. $x(t)$ is continuous and $\mathcal{F}_{t}$-adapted for $t \geq t_{0}-\tau$, where $\mathcal{F}_{t} \equiv \mathcal{F}_{t_{0}}$ for $t \in\left[t_{0}-\tau, t_{0}\right]$;

2. For every $T>t_{0}, \int_{t_{0}}^{T}\left|f\left(x_{t}, t\right)\right| d t<\infty$ a.s. and $\int_{t_{0}}^{T}\left|g\left(x_{t}, t\right)\right| d t<\infty$ a.s.;

3. For every $T>t_{0}$

$$
x(T)-D\left(x_{T}\right)=x\left(t_{0}\right)-D\left(x_{t_{0}}\right)+\int_{t_{0}}^{T} f\left(x_{s}, s\right) d s+\int_{t_{0}}^{T} g\left(x_{s}, s\right) \xi(s) d s
$$

A solution $x(t)$ to system (2) is said to be unique if any other solution $\bar{x}(t)$ is indistinguishable from $x(t)$.

To analyze the existence and uniqueness of solution to system (2), we impose the following assumptions.

Assumption A2 Both $f$ and $g$ are piecewise continuous in $t$ and satisfy the Lipschitz condition in $x$, i.e., there exists a constant $K>0$ such that

$$
|f(\phi, t)-f(\varphi, t)|+|g(\phi, t)-g(\varphi, t)| \leq K\|\phi-\varphi\|
$$

for all $t \geq t_{0}$ and $\forall \varphi, \phi \in C\left([-\tau, 0] ; R^{n}\right)$.

Assumption A3 There exists a constant $L>0$ such that

$$
|f(\phi, t)|+|g(\phi, t)| \leq L(1+\|\phi\|)
$$

for all $t \geq t_{0}$ and $\forall \phi \in C\left([-\tau, 0] ; R^{n}\right)$.

Assumption A4 There exists $\kappa \in(0,1)$ such that, for all $\varphi, \phi \in C\left([-\tau, 0] ; R^{n}\right)$,

$$
|D(\phi)-D(\varphi)| \leq \kappa\|\phi-\varphi\| .
$$

\section{The existence and uniqueness theorem}

Let us now establish the existence and uniqueness of solutions to system (2).

Theorem 1 Under Assumptions A1-A4, the neutral random functional nonlinear system (2) has a unique solution. 
Proof Uniqueness. Let $x(t)$ and $\bar{x}(t)$ be the two solutions of system (2) with initial data $x_{t_{0}}=\bar{x}_{t_{0}}$. By Assumptions A2 and A4, we have

$$
\begin{aligned}
& |x(t)-\bar{x}(t)| \\
& \quad \leq\left|D\left(x_{t}\right)-D\left(\bar{x}_{t}\right)\right|+\int_{t_{0}}^{t}\left|f\left(x_{s}, s\right)-f\left(\bar{x}_{s}, s\right)\right| d s+\int_{t_{0}}^{t}\left|g\left(x_{s}, s\right)-g\left(\bar{x}_{s}, s\right)\right||\xi(s)| d s \\
& \quad \leq \kappa\left\|x_{t}-\bar{x}_{t}\right\|+K \int_{t_{0}}^{t}\left\|x_{s}-\bar{x}_{s}\right\| d s+K \int_{t_{0}}^{t}\left\|x_{s}-\bar{x}_{s}\right\||\xi(s)| d s \\
& \quad \leq \kappa \sup _{t_{0}-\tau \leq s \leq t}|x(s)-\bar{x}(s)|+K \int_{t_{0}}^{t} \sup _{t_{0}-\tau \leq r \leq s}|x(r)-\bar{x}(r)|(1+|\xi(s)|) d s .
\end{aligned}
$$

This implies

$$
\begin{aligned}
& \sup _{t_{0} \leq s \leq t}|x(s)-\bar{x}(s)| \\
& \quad \leq \kappa \sup _{t_{0} \leq s \leq t}|x(s)-\bar{x}(s)|+K \int_{t_{0}}^{t} \sup _{t_{0} \leq r \leq s}|x(r)-\bar{x}(r)|(1+|\xi(s)|) d s,
\end{aligned}
$$

therefore

$$
\sup _{t_{0} \leq s \leq t}|x(s)-\bar{x}(s)| \leq \frac{K}{1-\kappa} \int_{t_{0}}^{t} \sup _{t_{0} \leq r \leq s}|x(r)-\bar{x}(r)|(1+|\xi(s)|) d s .
$$

Applying Gronwall's inequality yields that $x(t)=\bar{x}(t)$, a.s. for all $t \geq t_{0}$. The uniqueness is proved.

Existence. The proof of existence is divided into the following two steps.

Step 1. Define $x_{t_{0}}^{0}=\eta$ and $x^{0}(t)=\eta(0)$ for $t \geq t_{0}$. For each $n=1,2, \ldots$, set $x_{t_{0}}^{n}=\eta$ and define

$$
x^{n}(t)-D\left(x_{t}^{n-1}\right)=\eta(0)-D(\eta)+\int_{t_{0}}^{t} f\left(x_{s}^{n-1}, s\right) d s+\int_{t_{0}}^{t} g\left(x_{s}^{n-1}, s\right) \xi(s) d s .
$$

When $n=1$, we have

$$
x^{1}(t)-x^{0}(t)=D\left(x_{t}^{0}\right)-D(\eta)+\int_{t_{0}}^{t} f\left(x_{s}^{0}, s\right) d s+\int_{t_{0}}^{t} g\left(x_{s}^{0}, s\right) \xi(s) d s, \quad t \geq t_{0} .
$$

By Assumptions A2-A4, we have

$$
\begin{aligned}
\left|x^{1}(t)-x^{0}(t)\right| \leq & \left|D\left(x_{t}^{0}\right)-D(\eta)\right|+\int_{t_{0}}^{t}\left|f\left(x_{s}^{0}, s\right)\right| d s+\int_{t_{0}}^{t}\left|g\left(x_{s}^{0}, s\right)\right||\xi(s)| d s \\
\leq & \kappa\left\|x_{t}^{0}-x_{t_{0}}^{0}\right\|+K \int_{t_{0}}^{t}\left\|x_{s}^{0}-x_{t_{0}}^{0}\right\|(1+|\xi(s)|) d s \\
& +\int_{t_{0}}^{t}\left[\left|f\left(x_{t_{0}}^{0}, s\right)\right|+\left|g\left(x_{t_{0}}^{0}, s\right) \| \xi(s)\right|\right] d s \\
\leq & \kappa\left\|x_{t}^{0}-x_{t_{0}}^{0}\right\|+K \int_{t_{0}}^{t} \sup _{t_{0} \leq r \leq s}\left\|x_{r}^{0}-x_{t_{0}}^{0}\right\|(1+|\xi(s)|) d s \\
& +L \int_{t_{0}}^{t}(1+\|\eta\|)(1+|\xi(s)|) d s .
\end{aligned}
$$


Then, for any finite number $T>t_{0}$, we have

$$
\begin{aligned}
\sup _{t_{0} \leq t \leq T}\left|x^{1}(t)-x^{0}(t)\right| \leq & \kappa \sup _{t_{0} \leq t \leq T}\left\|x_{t}^{0}-x_{t_{0}}^{0}\right\|+K \int_{t_{0}}^{T} \sup _{t_{0} \leq r \leq s}\left\|x_{r}^{0}-x_{t_{0}}^{0}\right\|(1+|\xi(s)|) d s \\
& +L \int_{t_{0}}^{T}(1+\|\eta\|)(1+|\xi(s)|) d s \\
\leq & \sup _{t_{0} \leq t \leq T}\left\|x_{t}^{0}-x_{t_{0}}^{0}\right\|\left[\kappa+K \int_{t_{0}}^{T}(1+|\xi(s)|) d s\right] \\
& +L \int_{t_{0}}^{T}(1+\|\eta\|)(1+|\xi(s)|) d s .
\end{aligned}
$$

Note that

$$
\sup _{t_{0} \leq t \leq T}\left\|x_{t}^{0}-x_{t_{0}}^{0}\right\|=\sup _{t_{0}-\tau \leq t \leq T}\left\|x^{0}(t)-\eta\right\| \leq 2\|\eta\| .
$$

This together with (8) yields

$$
\sup _{t_{0} \leq t \leq T}\left|x^{1}(t)-x^{0}(t)\right| \leq 2 \kappa\|\eta\|+[2 K\|\eta\|+L(1+\|\eta\|)] \int_{t_{0}}^{T}(1+|\xi(s)|) d s:=C .
$$

On the other hand, we have

$$
\begin{aligned}
x^{n+1}(t)-x^{n}(t)= & D\left(x_{t}^{n}\right)-D\left(x_{t}^{n-1}\right)+\int_{t_{0}}^{t}\left[f\left(x_{s}^{n}, s\right)-f\left(x_{s}^{n-1}, s\right)\right] d s \\
& +\int_{t_{0}}^{t}\left[g\left(x_{s}^{n}, s\right)-g\left(x_{s}^{n-1}, s\right)\right] \xi(s) d s .
\end{aligned}
$$

By the same procedure as in the proof of the uniqueness and (9), we obtain

$$
\begin{aligned}
\sup _{t_{0} \leq t \leq T}\left|x^{n+1}(t)-x^{n}(t)\right| & \leq \sup _{t_{0} \leq t \leq T}\left|x^{n}(t)-x^{n-1}(t)\right|\left[\kappa+K \int_{t_{0}}^{T}(1+|\xi(s)|) d s\right] \\
& \leq C\left[\kappa+K \int_{t_{0}}^{T}(1+|\xi(s)|) d s\right]^{n} .
\end{aligned}
$$

Given $T-t_{0}$ is small enough such that $\kappa+K \int_{t_{0}}^{T}(1+|\xi(s)|) d s<1$, one can show from (10) that there is a solution to system (2) on $\left[t_{0}-\tau, T\right]$.

Step 2. Let $\sigma>0$ be sufficiently small for

$$
\kappa+K \int_{t_{0}}^{t_{0}+\sigma}(1+|\xi(s)|) d s<1
$$

by Step 1 , there is a solution to system (2) on $\left[t_{0}-\tau, t_{0}+\sigma\right]$ with initial data $x_{t_{0}}$. By Step 1 again, there is a solution to system (2) on $\left[t_{0}+\sigma, t_{0}+2 \sigma\right]$ with initial data $x_{t_{0}+\sigma}$. Repeating this procedure, we can derive that there is a solution to system (2) on $t \geq t_{0}-\tau$. The proof is complete. 
As in the classical SDE situation, the uniform Lipschitz Assumption A2 is somewhat restrictive in applications of the system, this motivates us to replace Assumption A2 with the local Lipschitz condition.

Assumption A2' Assume that for each $h>0$, there is a constant $K_{h}>0$ such that

$$
|f(\phi, t)-f(\varphi, t)|+|g(\phi, t)-g(\varphi, t)| \leq K_{h}\|\phi-\varphi\|
$$

for all $t \geq t_{0}$ and those $\varphi, \phi \in C\left([-\tau, 0] ; R^{n}\right)$ with $\|\varphi\| \vee\|\phi\| \leq h$.

Theorem 2 Under Assumptions A1, A2', A3, and A4, the neutral random functional nonlinear system (2) has a unique solution.

Following the same line of Theorem 2.3.4 in Mao [9], this theorem can be proved by a standard truncation procedure, so we omit it.

\section{Noise-to-state stability}

In this section, we consider a special but important class of system (2) of the form

$$
d[x(t)-\bar{D}(x(t-\tau))]=F(x(t), x(t-\tau), t) d t+G(x(t), x(t-\tau), t) \xi(t) d t, \quad t \geq t_{0}
$$

with initial data $x_{t_{0}}=\eta=\{\eta(\theta):-\tau \leq \theta \leq 0\} \in \mathcal{C}_{\mathcal{F}_{t_{0}}}^{b}\left([-\tau, 0] ; R^{n}\right)$, where $\bar{D}: R^{n} \rightarrow R^{n}, F$ : $R^{n} \times R^{n} \times R_{+} \rightarrow R^{n}$, and $G: R^{n} \times R^{n} \times R_{+} \rightarrow R^{n \times d}$ are Borel-measurable and the process $\xi(t)$ satisfies Assumption A1. We called (12) the neutral random delay nonlinear system.

For functions $\bar{D}, F$, and $G$, we impose the following assumptions.

Assumption A5 Both $F$ and $G$ satisfy the local Lipschitz condition. That is, for each $h>0$, there is a constant $K_{h}>0$ such that

$$
|F(x, y, t)-F(\bar{x}, \bar{y}, t)|+|G(x, y, t)-G(\bar{x}, \bar{y}, t)| \leq K_{h}(|x-\bar{x}|+|y-\bar{y}|)
$$

for all $t \geq t_{0}$ and $\forall x, \bar{x}, y, \bar{y} \in R^{n}$ with $|x| \vee|\bar{x}| \vee|y| \vee|\bar{y}| \leq h$.

Assumption A6 There exists a constant $L>0$ such that

$$
|F(x, y, t)|+|G(x, y, t)| \leq L(1+|x|+|y|)
$$

for all $t \geq t_{0}$ and $\forall x, y \in R^{n}$.

Assumption A7 There exists $\kappa \in(0,1)$ such that

$$
|\bar{D}(x)| \leq \kappa|x|
$$

for all $x \in R^{n}$.

In fact, system (12) can be written as system (2) by defining $f(\phi, t)=F(\phi, \phi(-\tau), t)$ and $g(\phi, t)=G(\phi, \phi(-\tau), t)$ for $(\phi, t) \in C\left([-\tau, 0] ; R^{n}\right) \times R_{+}$. Note that Assumptions A5, A6, and A7 imply Assumptions A2', A3, and A4 respectively, so by Theorem 2, there exists a unique solution to system (12) on $\left[t_{0}-\tau, \infty\right)$. 
Definition 2 For $p>0$, the neutral nonlinear system (12) is said to be noise-to-state exponentially stable in the $p$ th moment, if there exist positive constants $c, \lambda$ and a class $\mathcal{K}$ function $\gamma(\cdot)$ such that, for all $t \geq t_{0}$ and $x_{t_{0}}=\eta \in \mathcal{C}_{\mathcal{F}_{t_{0}}}^{b}\left([-\tau, 0] ; R^{n}\right)$,

$$
E|x(t)|^{p} \leq c E\|\eta\|^{p} e^{-\lambda\left(t-t_{0}\right)}+\gamma\left(\sup _{t_{0} \leq s \leq t} E|\xi(s)|^{2}\right) .
$$

When $p=2$, we also say that it is noise-to-state exponentially stable in the mean square.

The following lemma is a combination of Lemmas 4.3 and 4.5 in Kolmanovskii et al. [11].

Lemma 1 Let $p \geq 1$ and Assumption A7 hold. Then, for all $x, y \in R^{n}$,

$$
|x-\bar{D}(y)|^{p} \leq(1+\kappa)^{p-1}\left(|x|^{p}+\kappa|y|^{p}\right), \quad|x|^{p} \leq \kappa|y|^{p}+\frac{|x-\bar{D}(y)|^{p}}{(1-\kappa)^{p-1}} .
$$

Lemma 2 Let Assumptions A5-A7 hold. Suppose there exist positive constants $c_{1}, c_{2}, \alpha_{1}$, $\alpha_{2}, \alpha_{3}, d$ with $\alpha_{1}>\alpha_{2}$ and a $\mathcal{C}^{1}$ function $V(x, t): R^{n} \times\left[t_{0}, \infty\right) \rightarrow R_{+}$such that, for $p \geq 1$ and $\forall t \geq t_{0}, x, y \in R^{n}$,

$$
c_{1}|x|^{p} \leq V(x, t) \leq c_{2}|x|^{p}
$$

and

$$
\frac{\partial v}{\partial t}+\frac{\partial v}{\partial x} F(x, y, t)+d\left|\frac{\partial v}{\partial x} G(x, y, t)\right|^{2} \leq-\alpha_{1}|x|^{p}+\alpha_{2}|y|^{p}+\alpha_{3} .
$$

Moreover, there is a constant $\kappa \in(0,1)$ such that

$$
|\bar{D}(y)| \leq \kappa|y|, \quad \forall y \in R^{n} .
$$

Let $\bar{\lambda}$ be the unique solution to the equation

$$
\alpha_{1}-\lambda c_{2}(1+\kappa)^{p-1}-e^{\lambda \tau}\left(\alpha_{2}+\kappa \lambda c_{2}(1+\kappa)^{p-1}\right)=0 .
$$

If $\lambda \in(0, \bar{\lambda}]$, then for all $t \geq t_{0}$ and any initial data $\{\eta(\theta):-\tau \leq \theta \leq 0\}=\eta \in$ $\mathcal{C}_{\mathcal{F}_{t_{0}}}^{b}\left([-\tau, 0] ; R^{n}\right)$, it holds that

$$
e^{\lambda t} E|x(t)-\bar{D}(x(t-\tau))|^{p} \leq C_{\lambda} E\|\eta\|^{p}+\frac{\alpha_{3}}{\lambda c_{1}} e^{\lambda t}+\frac{1}{4 c_{1} d} e^{\lambda t} \sup _{t_{0} \leq s \leq t} E|\xi(s)|^{2},
$$

where $C_{\lambda}:=\frac{1}{c_{1}}\left[c_{2} e^{\lambda t_{0}}(1+\kappa)^{p}+\tau e^{\lambda \tau}\left(\alpha_{2}+\kappa \lambda c_{2}(1+\kappa)^{p-1}\right)\right]$.

Proof For $t \geq t_{0}$, let $\tilde{x}(t):=x(t)-\bar{D}(x(t-\tau))$, by Young's inequality and (16), we have

$$
\begin{aligned}
& \frac{d e^{\lambda t} V(\tilde{x}(t), t)}{d t} \\
& =\lambda e^{\lambda t} V(\tilde{x}(t), t)+e^{\lambda t}\left[V_{t}(\tilde{x}(t), t)+V_{x}(\tilde{x}(t), t) f(x(t), x(t-\tau), t)\right.
\end{aligned}
$$




$$
\begin{aligned}
& \left.+V_{x}(\tilde{x}(t), t) g(x(t), x(t-\tau), t) \xi(t)\right] \\
\leq & \lambda e^{\lambda t} V(\tilde{x}(t), t)+e^{\lambda t}\left[V_{t}(\tilde{x}(t), t)+V_{x}(\tilde{x}(t), t) f(x(t), x(t-\tau), t)\right] \\
& +d e^{\lambda t}\left|V_{x}(\tilde{x}(t), t) g(x(t), x(t-\tau), t)\right|^{2}+\frac{1}{4 d} e^{\lambda t}|\xi(t)|^{2} \\
\leq & \lambda e^{\lambda t} V(\tilde{x}(t), t)+e^{\lambda t}\left[-\alpha_{1}|x(t)|^{p}+\alpha_{2}|x(t-\tau)|^{p}+\alpha_{3}\right]+\frac{1}{4 d} e^{\lambda t}|\xi(t)|^{2} .
\end{aligned}
$$

Taking integrals and then expectations on both sides of the above, together with (15) and (17), we get

$$
\begin{aligned}
e^{\lambda t} E V(\tilde{x}(t), t) & \\
\leq & e^{\lambda t_{0}} E V\left(\tilde{x}\left(t_{0}\right), t_{0}\right)+E \int_{t_{0}}^{t} e^{\lambda s}\left[\lambda V(\tilde{x}(s), s)-\alpha_{1}|x(s)|^{p}\right. \\
& \left.+\alpha_{2}|x(s-\tau)|^{p}+\alpha_{3}\right] d s+\frac{1}{4 d} E \int_{t_{0}}^{t} e^{\lambda s}|\xi(s)|^{2} d s \\
\leq & c_{2} e^{\lambda t_{0}} E\left|x\left(t_{0}\right)-\bar{D}\left(x\left(t_{0}-\tau\right)\right)\right|^{p}+E \int_{t_{0}}^{t} e^{\lambda s}\left[\lambda c_{2}|\tilde{x}(s)|^{p}-\alpha_{1}|x(s)|^{p}\right. \\
& \left.+\alpha_{2}|x(s-\tau)|^{p}+\alpha_{3}\right] d s+\frac{1}{4 d} E \int_{t_{0}}^{t} e^{\lambda s}|\xi(s)|^{2} d s \\
\leq & c_{2} e^{\lambda t_{0}}(1+\kappa)^{p} E\|\eta\|^{p}+E \int_{t_{0}}^{t} e^{\lambda s}\left[\lambda c_{2}(1+\kappa)^{p-1}\left(|x(s)|^{p}+\kappa|x(s-\tau)|^{p}\right)\right. \\
& \left.-\alpha_{1}|x(s)|^{p}+\alpha_{2}|x(s-\tau)|^{p}+\alpha_{3}\right] d s+\frac{1}{4 d} E \int_{t_{0}}^{t} e^{\lambda s}|\xi(s)|^{2} d s \\
\leq & c_{2} e^{\lambda t_{0}}(1+\kappa)^{p} E\|\eta\|^{p}+\frac{\alpha_{3}}{\lambda} e^{\lambda t}+E \int_{t_{0}}^{t} e^{\lambda s}\left(-\alpha_{1}+\lambda c_{2}(1+\kappa)^{p-1}\right)|x(s)|^{p} d s \\
& +\left[\alpha_{2}+\kappa \lambda c_{2}(1+\kappa)^{p-1}\right] E \int_{t_{0}}^{t} e^{\lambda s}|x(s-\tau)|^{p} d s+\frac{1}{4 d} E \int_{t_{0}}^{t} e^{\lambda s}|\xi(s)|^{2} d s .
\end{aligned}
$$

However,

$$
E \int_{t_{0}}^{t} e^{\lambda s}|x(s-\tau)|^{p} d s \leq e^{\lambda \tau}\left(\tau E\|\eta\|^{p}+E \int_{t_{0}}^{t} e^{\lambda s}|x(s)|^{p} d s\right) .
$$

Substituting this into (19), we have

$$
\begin{aligned}
& e^{\lambda t} E V(\tilde{x}(t), t) \\
& \leq\left[c_{2} e^{\lambda t_{0}}(1+\kappa)^{p}+\tau e^{\lambda \tau}\left(\alpha_{2}+\kappa \lambda c_{2}(1+\kappa)^{p-1}\right) E\|\eta\|^{p}\right]+\frac{\alpha_{3}}{\lambda} e^{\lambda t} \\
& \quad-H(\lambda) E \int_{t_{0}}^{t} e^{\lambda s}|x(s)|^{p} d s+\frac{1}{4 d} E \int_{t_{0}}^{t} e^{\lambda s}|\xi(s)|^{2} d s,
\end{aligned}
$$

where $H(\lambda):=\alpha_{1}-\lambda c_{2}(1+\kappa)^{p-1}-e^{\lambda \tau}\left(\alpha_{2}+\kappa \lambda c_{2}(1+\kappa)^{p-1}\right)$. It is obvious that $H(\lambda)$ is continuous and mono-decreasing in $\lambda$. For $\alpha_{1}>\alpha_{2}$, and from the zero theorem, there exists a unique positive root $\bar{\lambda}$ to the equation $H(\lambda)=0$. Thus, for $\lambda \in(0, \bar{\lambda}]$, assertion (18) follows from (21) directly. 
Based on the above two lemmas, we can now state the main result of this section.

Theorem 3 Let the assumptions of Lemma 2 hold and $\alpha_{3}=0$. Let $\lambda \in\left(0, \bar{\lambda} \wedge \frac{1}{2 \tau} \log \left(\frac{1}{\kappa}\right)\right]$, then for any initial data $\{\eta(\theta):-\tau \leq \theta \leq 0\}=\eta \in \mathcal{C}_{\mathcal{F}_{t_{0}}}^{b}\left([-\tau, 0] ; R^{n}\right)$, the neutral random delay nonlinear system (12) is noise-to-state exponentially stable in the pth moment.

Proof For $\lambda \in\left(0, \bar{\lambda} \wedge \frac{1}{2 \tau} \log \left(\frac{1}{\kappa}\right)\right]$, by Lemmas 1 and 2 , we have

$$
\begin{aligned}
& e^{\lambda t} E|x(t)|^{p} \\
& \quad \leq \kappa e^{\lambda t} E|x(t-\tau)|^{p}+\frac{1}{(1-\kappa)^{p-1}} e^{\lambda t} E|x(t)-D(x(t-\tau))|^{p} \\
& \quad \leq \sqrt{\kappa} e^{\lambda(t-\tau)} E|x(t-\tau)|^{p}+\frac{1}{(1-\kappa)^{p-1}}\left[C_{\lambda} E\|\eta\|^{p}+\frac{1}{4 c_{1} d} e^{\lambda t} \sup _{t_{0} \leq s \leq t} E|\xi(s)|^{2}\right],
\end{aligned}
$$

where $C_{\lambda}$ is the same as defined in (18). Thus, we have

$$
\begin{aligned}
\sup _{t_{0} \leq s \leq t} e^{\lambda s} E|x(s)|^{p} \leq & \sqrt{\kappa}\left(E\|\eta\|^{p}+\sup _{t_{0} \leq s \leq t} e^{\lambda s} E|x(s)|^{p}\right) \\
& +\frac{1}{(1-\kappa)^{p-1}}\left[C_{\lambda} E\|\eta\|^{p}+\frac{1}{4 c_{1} d} e^{\lambda t} \sup _{t_{0} \leq s \leq t} E|\xi(s)|^{2}\right] .
\end{aligned}
$$

Therefore

$$
\begin{aligned}
\sup _{t_{0} \leq s \leq t} e^{\lambda s} E|x(s)|^{p} \leq & {\left[\frac{\sqrt{\kappa}}{1-\sqrt{\kappa}}+\frac{C_{\lambda}}{(1-\sqrt{\kappa})(1-\kappa)^{p-1}}\right] E\|\eta\|^{p} } \\
& +\frac{1}{4 c_{1} d(1-\sqrt{\kappa})(1-\kappa)^{p-1}} e^{\lambda t} \sup _{t_{0} \leq s \leq t} E|\xi(s)|^{2} .
\end{aligned}
$$

In particular,

$$
\begin{aligned}
E|x(t)|^{p} \leq & {\left[\frac{\sqrt{\kappa}}{1-\sqrt{\kappa}}+\frac{C_{\lambda}}{(1-\sqrt{\kappa})(1-\kappa)^{p-1}}\right] e^{-\lambda t} E\|\eta\|^{p} } \\
& +\frac{1}{4 c_{1} d(1-\sqrt{\kappa})(1-\kappa)^{p-1}} \sup _{t_{0} \leq s \leq t} E|\xi(s)|^{2},
\end{aligned}
$$

i.e., system (12) is noise-to-state exponentially stable in the $p$ th moment.

\section{An example}

In this section, an example is given to illustrate our main results.

Example 1 Let $\xi(t)$ be a one-dimensional stochastic process satisfying Assumption A1. Consider the following neutral nonlinear random system:

$$
d[x(t)-k x(t-\tau)]=\left[\beta_{1} x(t)+\beta_{2} x(t-\tau)\right] d t+\beta_{3} \sin x(t-\tau) \xi(t) d t
$$

with initial data $x_{t_{0}}=\eta=\{\eta(\theta):-\tau \leq \theta \leq 0\} \in \mathcal{C}_{\mathcal{F}_{t_{0}}}^{b}([-\tau, 0] ; R)$. 
Let $V(x, t)=x^{2}$, then (15) holds true for $c_{1}=0.5, c_{2}=1$, and $p=2$. For $\beta_{1}<0$, with the help of the elementary inequality $2 a b \leq a^{2}+b^{2}$, one can easily check that

$$
\begin{aligned}
\frac{\partial v}{\partial t} & +\frac{\partial v}{\partial x} F(x, y, t)+d\left|\frac{\partial v}{\partial x} G(x, y, t)\right|^{2} \\
& =2 \beta_{1} x^{2}+2 \beta_{2} x y-2 k \beta_{1} x y-2 k \beta_{2} y^{2}+4 d \beta_{3}^{2} \sin ^{2} y\left(x^{2}-2 k x y+k^{2} y^{2}\right) \\
& \leq\left[(2-k) \beta_{1}+\beta_{2}+4 d \beta_{3}^{2}(1+k)\right] x^{2}+\left[(1-2 k) \beta_{2}-k \beta_{1}+4 d \beta_{3}^{2}\left(k+k^{2}\right)\right] y^{2} .
\end{aligned}
$$

Set $k=0.1, \beta_{1}=-1, \beta_{2}=0.5, \beta_{3}=0.5$, and $d=0.1$, we have

$$
(2-k) \beta_{1}+\beta_{2}+4 d \beta_{3}^{2}(1+k)=-1.29
$$

and

$$
(1-2 k) \beta_{2}-k \beta_{1}+4 d \beta_{3}^{2}\left(k+k^{2}\right)=0.511
$$

By Theorem 3, system (22) is noise-to-state exponentially stable in the mean square.

\author{
Acknowledgements \\ We are deeply grateful to the three anonymous referees and the editor for their careful reading, valuable comments, and \\ correcting some errors, which have greatly improved the quality of the paper.
}

\title{
Funding
}

The work is supported by the Natural Science Foundation of Shandong Province (ZR2017MA015) and the National Natural Science Foundation of China $(11871076,71672166)$.

\section{Competing interests}

The author declares that they have no competing interests.

\section{Authors' contributions}

WL contributed to the work totally, and he read and approved the final version of the manuscript.

\section{Publisher's Note}

Springer Nature remains neutral with regard to jurisdictional claims in published maps and institutional affiliations.

Received: 6 May 2018 Accepted: 1 August 2018 Published online: 05 September 2018

\section{References}

1. Arnold, L.: Stochastic Differential Equations: Theory and Applications. Wiley, New York (1972)

2. Friedman, A.: Stochastic Differential Equations and Applications, vol. 2. Academic Press, New York (1976)

3. Mohammed, S.E.A.: Stochastic Functional Differential Equations. Pitman, Boston (1984)

4. Has'minskii, R.Z.: Stochastic Stability of Differential Equations. Sijthoff \& Noordhoff, Alphen (1981)

5. Mao, X:: Stability of stochastic differential equations with Markovian switching. Stoch. Process. Appl. 79, 45-67 (1999)

6. Yuan, C., Mao, X.: Stochastic Differential Equations with Markovian Switching. Imperial College Press, London (2006)

7. Hale, J.K., Lunel, S.M.V.: Introduction to Functional Differential Equations. Springer, New York (1993)

8. Kolmanovskii, V.B., Nosov, V.R.: Stability and Periodic Modes of Control Systems with Aftereffect. Nauka, Moscow (1981)

9. Mao, X.: Stochastic Differential Equations and Their Applications. Horwood, Chichester (1997)

10. Mao, X.: Asymptotic properties of neutral stochastic differential delay equations. Stoch. Stoch. Rep. 68, 273-295 (2000)

11. Kolmanovskii, V., Koroleva, N., Maizenberg, T., Mao, X.: Neutral stochastic differential delay equations with Markovian switching. Stoch. Anal. Appl. 21, 819-847 (2003)

12. Mao, X., Shen, Y., Yuan, C.: Almost surely asymptotic stability of neutral stochastic differential delay equations with Markovian switching. Stoch. Process. Appl. 118, 1385-1406 (2008)

13. Xie, Y., Zhang, C.: Asymptotical boundedness and moment exponential stability for stochastic neutral differential equations with time-variable delay and Markovian switching. Appl. Math. Lett. 70, 46-51 (2017)

14. Wu, Z:: Stability criteria of random nonlinear systems and their applications. IEEE Trans. Autom. Control 60, 1038-1049 (2015)

15. Zhang, H., Xia, Y., Wu, Z:: Noise-to-state stability of random switched systems and its applications. IEEE Trans. Autom. Control 61, 1607-1612 (2016) 
16. Krstic, M., Deng, H.: Stability of Nonlinear Uncertain Systems. Springer, New York (1998)

17. Deng, H., Krstic, M., Williams, R.J.: Noise-to-state stability of random switched systems and its applications. IEEE Trans. Autom. Control 46, 1237-1253 (2001)

18. Zhang, D., Wu, Z., Sun, X., Shi, P.: Noise-to state stability for random affine systems with state-dependent switching. In: Proceeding of the 11th World Congress on Intelligent Control and Automation, pp. 2191-2195 (2014)

19. Zhang, D., Wu, Z., Sun, X., Wang, W.: Noise-to-state stability for a class of random systems with state-dependent switching. IEEE Trans. Autom. Control 61, 3164-3170 (2016)

Submit your manuscript to a SpringerOpen ${ }^{\circ}$ journal and benefit from:

- Convenient online submission

$\checkmark$ Rigorous peer review

- Open access: articles freely available online

- High visibility within the field

Retaining the copyright to your article

Submit your next manuscript at $\boldsymbol{\nabla}$ springeropen.com 